\title{
Vortex Tube: A Comparison of Experimental and CFD Analysis Featuring Different RANS Models
}

\author{
Radomír Chýlek ${ }^{1, *}$, Ladislav Šnajdárek ${ }^{1}$, and Jiři Pospíšil ${ }^{1}$ \\ ${ }^{1}$ Brno University of Technology, Faculty of Mechanical Engineering, Dept. of Power Eng., Technická \\ 2896/2 Brno, Czech Republic
}

\begin{abstract}
The Ranque-Hilsch vortex tube represents a device for both cooling and heating applications. It uses compressed gas as drive medium. The temperature separation is affected by fluid flow behaviour inside the tube. It has not been sufficiently examined in detail yet and has the potential for further investigation. The aim of this paper is to compare results of numerical simulations of the vortex tube with obtained experimental data. The numerical study was using computational fluid dynamics (CFD), namely computational code STAR-CCM+. For the numerical study, a threedimensional geometry model, and various turbulence physics models were used. For the validation of carried out calculations, an experimental device of the vortex tube of identical geometrical and operating conditions was created and tested. The numerical simulation results have been obtained for five different turbulence models, namely Standard k- $\varepsilon$, Realizable $\mathrm{k}-\varepsilon$, Standard k- $\omega$, SST k- $\omega$ and Reynolds stress model (RSM), were compared with experimental results. The most important evaluation factor was the temperature field in the vortex tube. All named models of turbulence were able to predict the general flow behaviour in the vortex tube with satisfactory precision. Standard k- $\varepsilon$ turbulence model predicted temperature distribution in the best accordance with the obtained experimental data.
\end{abstract}

\section{Introduction}

Cooling and heating performance of a Ranque-Hilsch vortex tube is a subject of research nearly a century after it was accidentally invented by Ranque. This simple and compact device operates without any moving mechanical parts and simultaneously separates a pressurized gas into cold and hot fractions. A simplified model of the counter-flow vortex tube with displayed streamlines is shown in Figure 1. Experimental, analytical and numerical studies have been developed for better understanding of the problem. These studies were focused on gas thermal separation and energy transformations within the vortex tube, with a target to achieve better cooling and heating effects. In contrast with the simplicity of the device itself, the observed energy and flow separation processes are very challenging to describe it analytically or numerically.

\footnotetext{
* Corresponding author: radomir.chylek@,vut.cz
} 


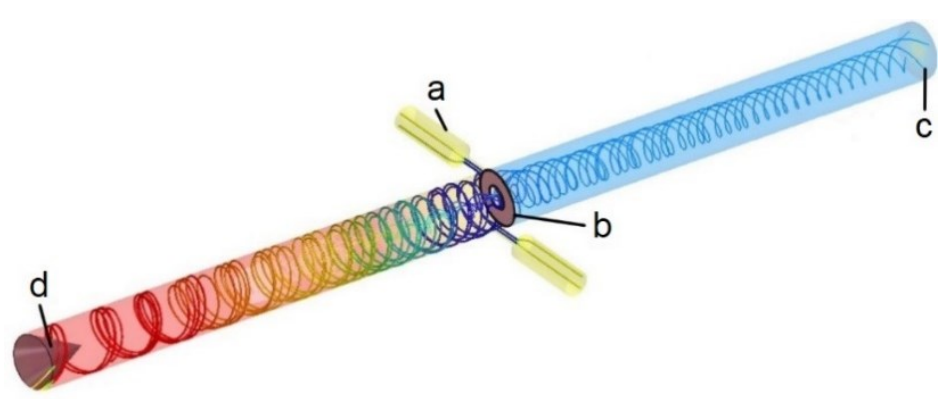

Fig. 1. Schematic model of the vortex tube; a-tangential inlet nozzle, b-cold exit orifice, c-cold outlet, d-hot control valve and hot outlet.

There is no general agreement on the theory of such a complex process. However, radial pressure gradient, generated by gas entering the tube after leaving nozzles, is considered to be the main reason for the occurring temperature and flow separation effects. A rapidly spinning vortex of the air is created, when air expands after passing the nozzles, at the outer diameter of a hot section of the vortex tube. A fraction of the air, named as a hot fraction, leaves the hot section at the hot end, after passing through a control valve. Remaining second fraction of the air is due to the control valve forced to move in an opposite direction to the hot fraction. It forms an inner vortex which is heading to the opposite side than the outer vortex of the hot fraction. Gas of the inner flow transfers heat energy to the outer flow when passing the hot section. Then the cooled inner flow passes through the cold exit orifice, enters a cold section and leaves the vortex tube colder as a cold fraction. Difference between temperature of the air at inlet $T_{I N}$ and at the cold end $T_{C}$ is given as:

$$
\Delta T_{C}=\left|T_{I N}-T_{C}\right|
$$

Hot end temperature $T_{H}$ difference is defined similarly, as:

$$
\Delta T_{H}=\left|T_{I N}-T_{H}\right|
$$

All numerical studies of the vortex tube are bound by a selection of a suitable turbulent model. The selected numerical model must describe a continuum of the gas moving through the vortex tube as accurately as possible in terms of flow description, energy separation and temperature and pressure field. In the present state of development of computing power, a Computational Fluid Dynamics (CFD) numerical analysis can be used for fully threedimensional simulations of the vortex tube including complex turbulent and physics models.

A large variety of both RANS and LES turbulent models was used for two-dimensional simulations, or partially and fully three-dimensional simulations, to produce results of differing accuracy when compared to those obtained by an experiment. However, from the previous research it is not clear which turbulent model has achieved the best agreement with experimental results in terms of temperature fields. Models used most frequently were twoequation turbulence models k- $\varepsilon$ Standard and k- $\varepsilon$ Realizable, Reynolds stress model (RSM) and Large eddy simulation model (LES) with the Smagorinsky-Lilly variant. Numerical studies mostly focused on the optimization of the vortex tube parameters and enhancing its performance using some of these turbulence models. A list of previously used turbulent models for selected numerical analysis of the vortex tube is shown in Table 1. 
Table 1 indicates that nowadays authors are turning to more demanding threedimensional models, either fully three-dimensional or with axis-symmetrical conditions. Different authors tried to apply Large Eddy Simulation (LES) and RSM models, which are computationally more expensive than two-equation RANS methods. The most commonly used turbulent model is Standard k- $\varepsilon$ model due to the accuracy of some reported results and lower computational costs.

Table 1. Summary of selected numerical studies on vortex tubes.

\begin{tabular}{|c|c|c|c|c|}
\hline Year & Name of the author & Geometry & Used turbulence model & Software \\
\hline 1999 & Fröhlingsdorf [1] & $2 \mathrm{D}$ & Keyes k- $\varepsilon$ & CFX \\
\hline 2005 & Aljuwayhel [2] & $2 \mathrm{D}$ & Standard k- $\varepsilon$, RNG k- $\varepsilon$ & Fluent \\
\hline 2006 & Eiamsa-ard [3] & $2 \mathrm{D}$ & Algebraic RSM (ASM) & TEFESS \\
\hline 2006 & Skye [4] & 2D & Standard k- $\varepsilon$, RNG k- $\varepsilon$ & Fluent \\
\hline 2008 & Behera [5] & $3 \mathrm{D}$ & RNG k- $\varepsilon$ & STAR-CD \\
\hline 2009 & Farouk [6] & $2 \mathrm{D}$ & LES & CFD-ACE+ \\
\hline 2010 & Shamsoddini [7] & $3 \mathrm{D}$ & RNG k- $\varepsilon$ & Fluent \\
\hline 2013 & Ouadha [8] & 3D & RSM & Fluent \\
\hline 2014 & Bovand [9] & $3 \mathrm{D}$ & RNG k- $\varepsilon$ & Fluent \\
\hline 2014 & Alekhin [10] & $3 \mathrm{D}$ & SAT-SST & CFX \\
\hline 2014 & Liu [11] & $3 \mathrm{D}$ & Realizable k- $\varepsilon$ & Fluent \\
\hline 2016 & Bianco [12] & $3 \mathrm{D}$ & RSM-LRR, LES & CFX \\
\hline 2017 & Thakare [13] & $2 \mathrm{D}$ & Standard $k-\varepsilon$ & Fluent \\
\hline
\end{tabular}

The following numerical studies are directly devoted to the problem of selecting a suitable turbulent model for the counter-flow vortex tubes. A two-dimensional axis-symmetrical model was used for various turbulence models and discretization schemes by Thakare [14]. It was found that the most suitable turbulence model was $k-\varepsilon$ Standard. It was observed that the deviation in the prediction of a total temperature of air in the cold region, as well as other parameters of the flow, were in an acceptable range using k- $\varepsilon$ Standard model.

Eimsa-ard et al. [15] used Algebraic Stress Model (ASM) and Standard k- $\varepsilon$ turbulence model for simulating the thermal separation. ASM had better agreement with experimental results.

Liu and Liu [11] compared numerical results of five turbulence models (SST, Standard

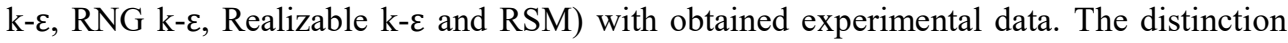
between experimental results and the numerical model was smallest using k- $\varepsilon$ Realizable and RSM models. Similar studies with similar results were conducted by Baghdad [16] and Dutta [17], but with different criteria for choosing the appropriate turbulent model.

Farouk et al. [18] carried out Large Eddy Simulation (LES) and observed that energy separation by LES was in better agreement with experiment than results obtained using k-E. However, LES under predicted the cold temperature drop.

Secchiaroli et al. [19] handled LES Smagorinsky sub-grid scale closure in the simulations of the vortex tube. They also employed RSM and RNG k- $\varepsilon$ models to compare temperature and velocity plots. It was found that RSM agrees with LES better than RNG k- $\varepsilon$ in case of temperature and velocity profiles.

In terms of a double-circuit vortex tube, Alekhin et al. [10] and Bianco et al. [12] investigated and compared Standard k- $\varepsilon$, RNG k- $\varepsilon$, RSM, SAS-SST and LES turbulent models for a more complex double-circuit vortex tube. It was found that RSM and LES turbulence models are the most suitable for this more complicated device.

However, several important factors can influence the choice of turbulence model for vortex tube numerical analysis. Mainly the selection of a fully three-dimensional model can play a key role. Choosing specific geometrical parameters of the tube and its inlet and outlet operating parameters should also be evaluated. 
The main aim of this work is to choose the most accurate turbulence model for the fully three-dimensional model of the vortex tube in terms of temperature distribution and flow separation within the tube. STAR-CCM+ computational code was used for the numerical study, and the selection of turbulence was directed to RANS-based turbulence models, specifically to two-equation RANS models and Reynolds Stress Transport model. To compare turbulent models, an experimental vortex tube of corresponding geometrical and operating conditions was created and analysed.

\section{Experimental study}

For comparison and validation of numerical simulations, the experimental device was set up. Its essential geometrical parameters are listed in Table 2. The same parameters were used in the numerical simulations. The material of the vortex tube casing was Polymethyl methacrylate-PMMA. Six tangential nozzles were extruded to the nozzle module of Polyoxymethylene (POM).

Table 2. Geometric parameters of the experimental vortex tube.

\begin{tabular}{|c|c|c|}
\hline Parameter & Value & Unit \\
\hline Hot section length & 360 & {$[\mathrm{~mm}]$} \\
\hline Cold section length & 200 & {$[\mathrm{~mm}]$} \\
\hline Number or inlet nozzles & 6 & {$[-]$} \\
\hline Vortex tube diameter & 24 & {$[\mathrm{~mm}]$} \\
\hline Cold exit orifice diameter & 8.5 & {$[\mathrm{~mm}]$} \\
\hline Hot control valve diameter & 23.4 & {$[\mathrm{~mm}]$} \\
\hline Nozzle diameter & 2 & {$[\mathrm{~mm}]$} \\
\hline
\end{tabular}

Pressurized air was supplied by the compressor, accumulated in the air tank and dried in the air dryer. Before the air enters the nozzles of the vortex tube, its pressure is regulated by the reducing valve. Temperature (thermocouple type $\mathrm{T}$, accuracy $\pm 0.25^{\circ} \mathrm{C}$ ), volume flow (accuracy $\pm 5 \%$ ), absolute pressure (accuracy $\pm 0.35 \%$ ) and relative pressure (accuracy $\pm 0.6 \%$ ) of the inlet air were measured. The volume flow of the cold fraction is measured at the cold end of the vortex tube by the anemometer (accuracy $\pm 1 \%$ ). The schematic diagram of the experimental setup is presented in Figure 2. The experimental setup of the vortex tube is shown in Figure 3.

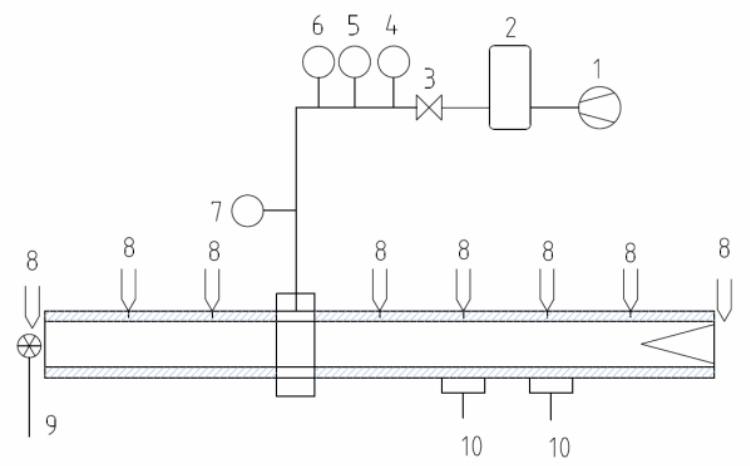

Fig. 2. Schematic diagram of the experimental setup. 1-compressor, 2-air tank and air dryer, 3-reducing valve, 4-relative pressure sensor, 5-thermocouple, 6-flowmeter, 7-absolute pressure sensor, 8 -type T thermocouples, 9-anemometer, 10-heat flux sensor. 


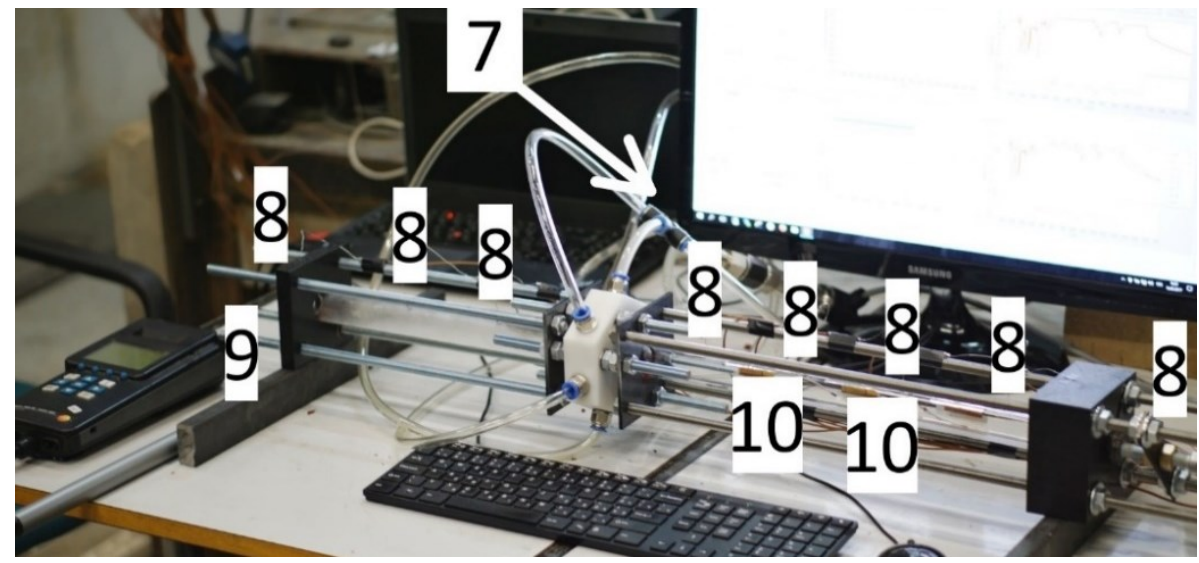

Fig. 3. The experimental setup of the vortex tube. 7-absolute pressure sensor, 8-type T thermocouples, 9-anemometer, 10-heat flux sensors.

The temperature of the cold and hot fraction is measured by type $\mathrm{T}$ thermocouples (accuracy $\pm 0.1 \%$ ) at both ends of the vortex tube. The thermocouples were also embedded into drilled holes of the tube casing at regular distances along the length of the tube and placed $1 \mathrm{~mm}$ from the inner surface of the vortex tube. Tube casing is also equipped with heat flux sensors (accuracy $\pm 3 \%$ ) at the hot section to monitor and determine the steady state of the measurement.

After the compressor pressurized the air tank to 8 bar, reducing valve was released until the desired relative pressure of 4 bar was reached for the inlet nozzles. The experimental setup is tested for any leakage in the system. Software LabVIEW and National Instruments instrumentation were used to monitor and regulate the system. The temperature of the air at the inlet is $20^{\circ} \mathrm{C}$. Cone control valve is centered and the distance between two created concentric circles is set to $0.3 \mathrm{~mm}$. The experimental observation requires $30-45 \mathrm{~min}$. until all temperature and heat flux sensors reported steady-state values.

\section{Numerical study}

\subsection{Geometry}

A fully three-dimensional model was used for the numerical simulations. Geometric parameters for the numerical model correspond with parameters of the experimental device in Table 2. Relaxation section was added beyond the hot end orifice to determine the temperature of the hot fraction. The geometry of the vortex tube is shown in Figure 4. 


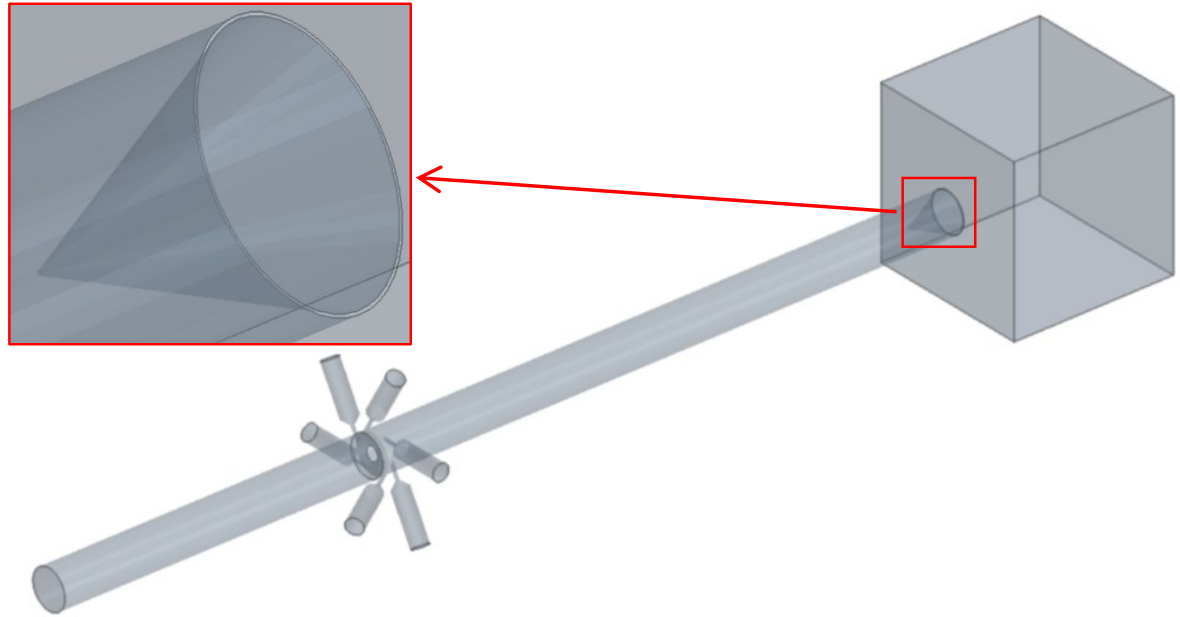

Fig. 4. The geometry of the vortex tube with the hot orifice in the detail.

\subsection{Mesh}

Automatic mesher integrated into STAR-CCM+ was used to create a partially structured mesh. The polyhedral mesher and the prism layer mesher modules were used. The resulting mesh contains about 8.2 million cells. The number of cells was slightly different according to the used turbulent module and its requirements for the boundary layer. A grid independence study of solution was conducted. For fewer than 1 million cells, the fully $3 \mathrm{D}$ simulation was unstable for some turbulence models. For 8 million to 13 million of grid cells, temperature values for hot and cold fraction were within a one-percent range for the individual turbulent model. The volumetric control refinements were used in the sections of higher pressure, higher temperature gradients, and with transonic velocity. Refinements were primarily used in the section of the inlet nozzles and near the hot end orifice. High velocity of air is reached near the nozzle inlets $(\mathrm{Ma}>0.8)$ and hot end refinements, see Figure 5.

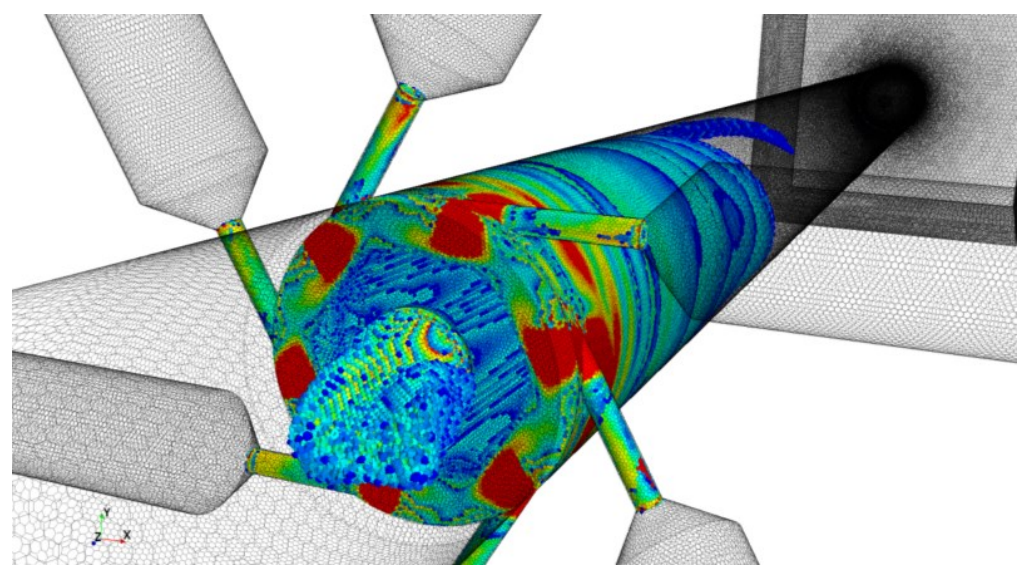

Fig. 5. High velocity of air at the nozzle inlets $(\mathrm{Ma}>0.8)$ and hot end refinement. 


\subsection{Governing equations}

The turbulent, compressible flow of real gas (Redlich-Kwong equation) was considered for the carried out numerical analysis. Governing equations of continuity, momentum, and energy and mass are:

$$
\begin{gathered}
\frac{\partial \rho}{\partial t}+\nabla \cdot(\rho \boldsymbol{v})=0 \\
\frac{\partial(\rho \boldsymbol{v})}{\partial t}+\rho(\boldsymbol{v} \cdot \nabla) \boldsymbol{v}=-\nabla p+\nabla \cdot \tau \\
\frac{\partial}{\partial t}(\rho H)+\nabla(\rho \boldsymbol{v} H)-\nabla\left(\frac{\lambda+\lambda_{t}}{c_{p}} \nabla h\right)=\frac{\partial p}{\partial t} \\
p=\frac{R T}{V-b_{1}}-\frac{a_{1}}{T^{0,5} V\left(V+b_{1}\right)}
\end{gathered}
$$

Where $\rho$ is air density, $H$ is total enthalpy, $h$ is static enthalpy, $p$ is static pressure, $\tau$ is stress tensor, $v$ is velocity, $\lambda$ is coefficient of molecular thermal conductivity, $c_{p}$ is heat capacity, $V$ is volume, $R$ is gas constant and constants $a_{l}$ and $b_{l}$ are defined as:

$$
\begin{aligned}
& a_{1}=0,42748 \frac{R^{2} T_{c r}^{2,5}}{p_{c r}} \\
& b_{1}=0,08664 \frac{R T_{c r}}{p_{c r}}
\end{aligned}
$$

Where $T_{C R}[\mathrm{~K}]$ is critical temperature, $p_{C R}$ is cirtical pressure $[\mathrm{Pa}]$ and Turbulent thermal conductivity $\lambda_{t}$ is calculated from turbulent Prandtl number $\operatorname{Pr}_{t}$ as:

$$
\frac{\lambda_{t}}{c_{p}}=\frac{\mu_{t}}{\operatorname{Pr}_{t}}
$$

Where the coefficient of turbulent dynamic viscosity $\mu_{t}$ is given by means of turbulence models (k- $\varepsilon, k-\omega$, RSM Linear Pressure Strain). Turbulent Prandtl number $P r_{t}$ was set to 0.8 .

Used RANS models offer the most economic approach for computing complex turbulent flows. RANS models simplify the problem of turbulence to the solution of two additional transport equations and introduce a turbulent viscosity to compute Reynolds stresses. One of the transported variable is the turbulent kinetic energy $k$ (it determines the energy of the turbulence), the second is for certain cases Turbulent disipation $\varepsilon$ (k- $\varepsilon$ Standard and k- $\varepsilon$ Realizable) or the Specific turbulent disipation $\omega$ (k- $\omega$ Standard or k- $\omega$ SST) and it determines the scale of turbulence. More complex RANS models that solves an individual equation for each of the six independent Reynolds Stresses directly are Reynolds Stress Models (RSM). 


\subsection{Boundary conditions}

Numerical simulation was initialized in a steady-state regime with the coupled solver. After it sufficiently advanced, it was shifted to an implicit unsteady regime. Reference pressure was set to $101325 \mathrm{~Pa}$. The initial static temperature was $20^{\circ} \mathrm{C}$. Boundary conditions are shown in Table 3.

Table 3. Boundary conditions for numerical models.

\begin{tabular}{|c|c|c|c|}
\hline Boundary & Quantity & Value & Unit \\
\hline \multirow{3}{*}{ Inlet nozzles } & Relative pressure & 4 & {$[\mathrm{bar}]$} \\
\cline { 2 - 4 } & Temperature & 20 & {$\left[{ }^{\circ} \mathrm{C}\right]$} \\
\cline { 2 - 4 } & Turbulence intensity & $5 \%$ & {$[-]$} \\
\hline Hot outlet & Relative pressure & 0 & {$[\mathrm{~mm}]$} \\
\hline Cold outlet & Relative pressure & 0 & {$[-]$} \\
\hline \multirow{2}{*}{ Walls } & Shear stress & No slip & {$[-]$} \\
\cline { 2 - 4 } & Thermal specification & Adiabatic & {$[-]$} \\
\hline
\end{tabular}

\subsection{Numerical solution}

All solutions were performed in STAR-CCM+ software. The coupled solver was used for all carried out calculations with turbulence model based on the hybrid LSQ-Gauss gradient method. Second-order upwind discretization scheme was selected. First-order temporal scheme (Euler Implicit) was chosen for transient calculations.

Simulations were executed on the cluster on sixteen processor cores arranged in a computational cluster. One transient simulation took from 10-30 days to simulate 0.050.2 second of the physical time. Calculation times were dependent on the turbulent model, convergence status and rate of stabilization of temperature field in the vortex tube. Time-step was set to values from $1.0 \mathrm{E}-6$ to $1.0 \mathrm{E}-5$ with the number of inner iterations from 5 to 10 . Residual values were kept below 1.0E-4 with this procedure. Temperatures at the hot and cold end were monitored in order to provide monitor-based judging criteria of convergence, together with the residual criterion.

\section{Results and discussion}

This section is dedicated to the validation of used numerical models. Results from individual numerical models were compared with obtained experimental data. The comparison was focused on the temperature distribution. Firstly, the total temperature distribution in the midplane of the tube is shown in Figure 6-10 for every turbulence model. Minimal and maximal range values of total temperature were varied based on maximum and minimum temperature achieved by the individual turbulence model. It was observed, that the general flow behaviour creates hot and cold sections in every case, not depending on the used turbulence model. Energy separation mechanism was therefore not affected by used turbulent model. However, the maximum and minimum temperature values were dependent on the turbulent model. 


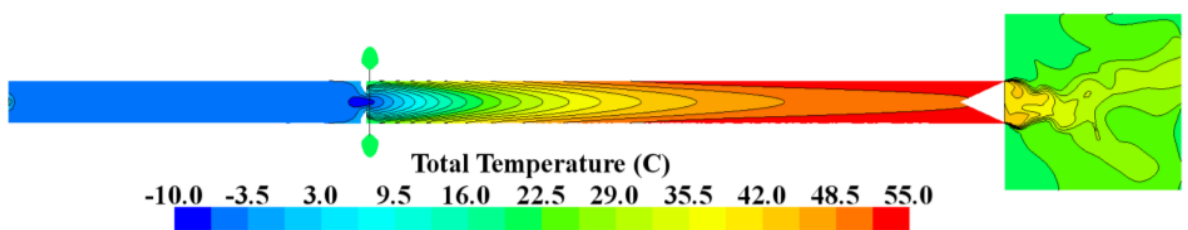

Fig. 6. Total temperature distribution for k- $\varepsilon$ Standard turbulence model.

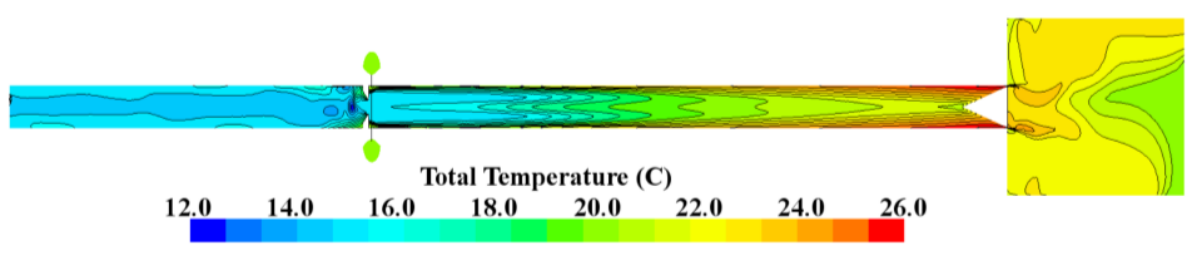

Fig. 7. Total temperature distribution for k- $\varepsilon$ Realizable turbulence model.

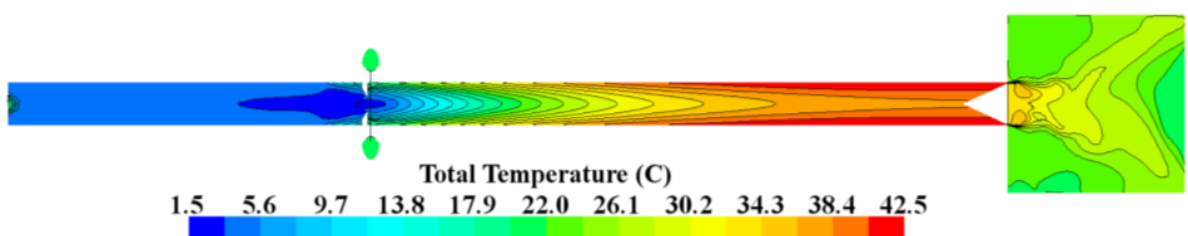

Fig. 8. Total temperature distribution for k- $\omega$ SST turbulence model.

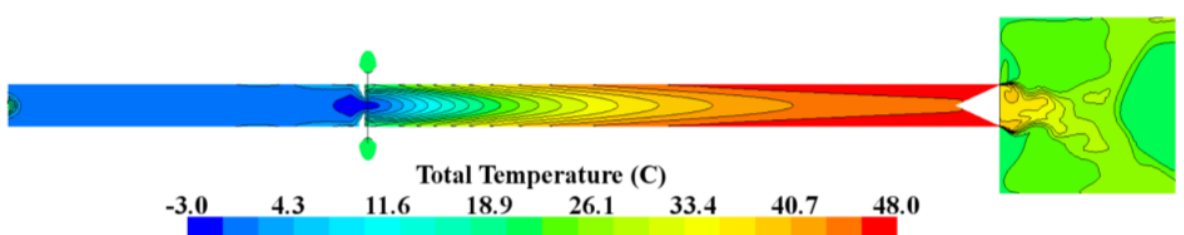

Fig. 9. Total temperature distribution for k- $\omega$ Standard (Wilcox) turbulence model.

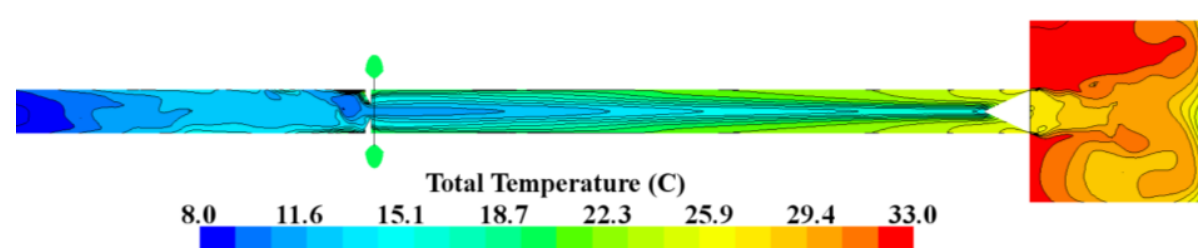

Fig. 10. Total temperature distribution for RSM turbulence model. 
In order to compare the values of total temperature of the hot and cold fraction, $\%$ cold and hot deviation is declared as:

$$
\begin{aligned}
& \%\left(\Delta T_{C}\right)=\left|\frac{\left(\Delta T_{C}\right)_{\exp }-\left(\Delta T_{C}\right)_{c f d}}{\left(\Delta T_{C}\right)_{\exp }}\right| \times 100 \\
& \%\left(\Delta T_{H}\right)=\left|\frac{\left(\Delta T_{H}\right)_{\exp }-\left(\Delta T_{H}\right)_{c f d}}{\left(\Delta T_{H}\right)_{\exp }}\right| \times 100
\end{aligned}
$$

These \% deviations between experimental and CFD total temperature values of hot and cold fraction are shown in Table 4.

\begin{tabular}{|c|c|c|c|c|}
\hline & $\mathbf{T}_{\mathbf{C}}$ & $\mathbf{T}_{\mathrm{H}}$ & $\%\left(\Delta T_{C}\right)$ & $\%\left(\Delta \mathrm{T}_{\mathrm{H}}\right)$ \\
\hline Experiment & -1.8 & 57.2 & {$[-]$} & {$[-]$} \\
\hline k- $\varepsilon$ Standard & -3.0 & 54.9 & 5.50 & 6.18 \\
\hline k- $\varepsilon$ Realizable & 15.4 & 25.3 & 78.90 & 85.75 \\
\hline$k-\omega$ SST & 4.6 & 41.0 & 29.36 & 43.54 \\
\hline k- $\omega$ Standard & 1.8 & 47.0 & 16.51 & 27.42 \\
\hline RSM & 8.1 & 32.3 & 45.41 & 66.94 \\
\hline
\end{tabular}

Table 4. \% deviations of the cold and hot end temperatures.

Temperatures along the tube were also compared in Figure 11. For the experiment, temperatures were measured by thermocouples located in drilled holes on the surface of the plastic tube in points 1-6, and with two thermocouples measuring temperature of the cold and hot fraction $\left(\mathrm{T}_{\mathrm{C}}\right.$ and $\left.\mathrm{T}_{\mathrm{H}}\right)$. Numerical values were gathered from a points at a distance of $1 \mathrm{~mm}$ from the wall.

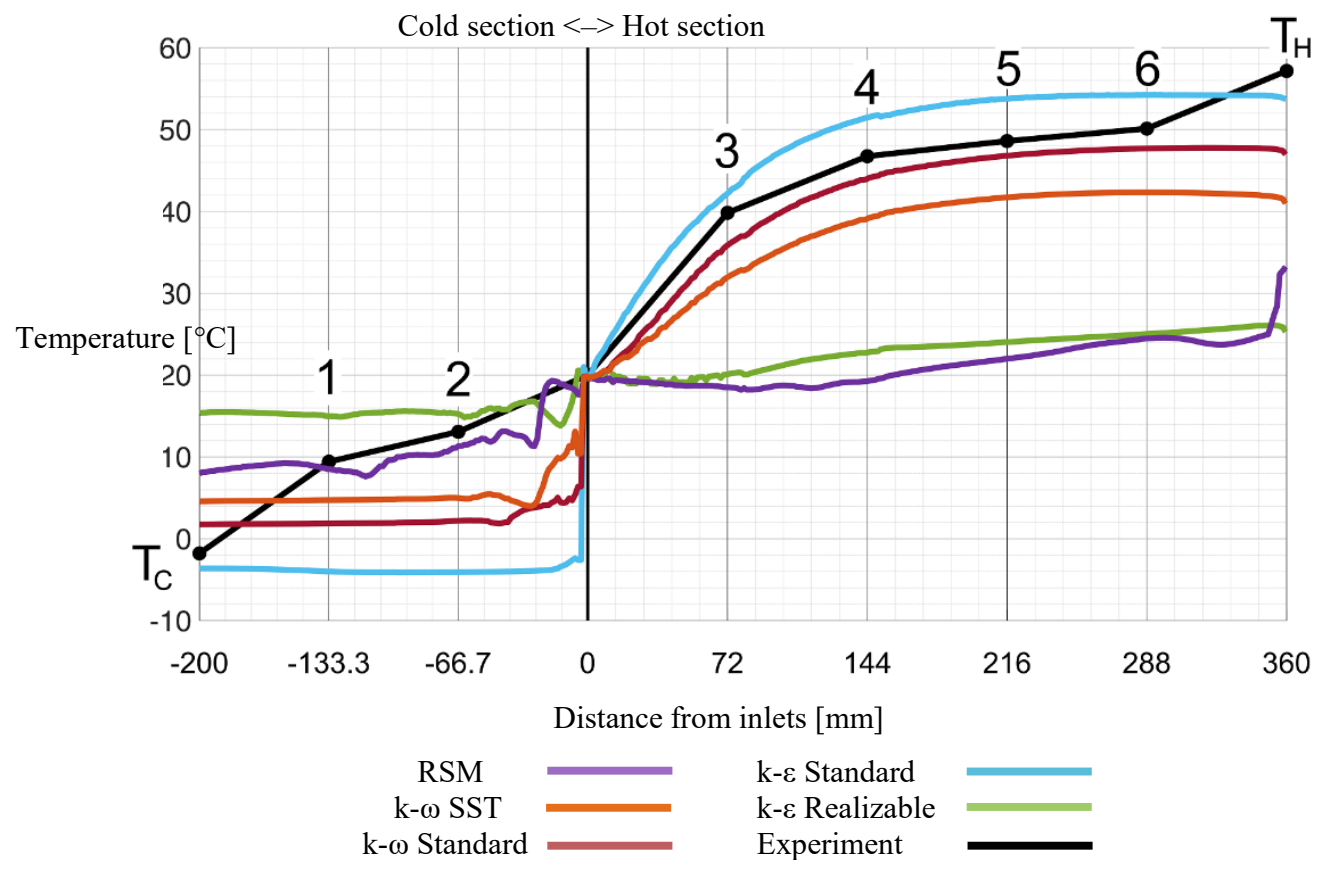

Fig. 11. Distribution of total temperatures. 
As can be observed from Table 4 and Figure 11, the smallest deviation was achieved with turbulence model k- $\varepsilon$ Standard. Both for the cold and hot fraction \% deviations. The greater discrepancy in points 1 and 2 in the Figure 11 was caused by heat conduction of the plastic tube used for the experimental study. Nothing suggests that the cold section causes the cold fraction to cool down more. For this reason, it can be assumed that the temperature of the air $\mathrm{T}_{\mathrm{C}}$ of the cold fraction is characteristic for the entire cold section.

\section{Conclusion}

For the numerical study, a three-dimensional turbulent real gas model was used. Numerical simulations were carried out in computational code STAR-CCM+. Simulation were using various turbulence models, namely: Standard k- $\varepsilon$, Realizable k- $\varepsilon$, Standard k- $\omega$, SST k- $\omega$ and RSM. Presented numerical models were validated and compared with the obtained experimental data. The following major conclusions were summarized:

The general temperature distribution, energy separation effects, and flow behaviour worked with every used turbulence model to some extent.

However, magnitudes of $\Delta \mathrm{T}_{\mathrm{C}}$ and $\Delta \mathrm{T}_{\mathrm{H}}$ were different depending on the used turbulence model in the numerical simulation.

Significant deviations in cold and hot fraction temperature differences of Realizable k$\varepsilon$ turbulence model were observed.

More demanding RSM model should produce more complex and accurate velocity fields thanks to the anisotropy and six more partial differential equations for each component of Reynolds stress tensor solved. However, it underpredicted magnitudes of $\Delta \mathrm{T}_{\mathrm{C}}$ and $\Delta \mathrm{T}_{\mathrm{H}}$ as compared with RANS models, except k- $\varepsilon$ Realizable model.

Models k- $\varepsilon$ Standard and k- $\omega$ Standard predicted temperatures of both fractions in the better accordance with the obtained experimental data than other models covered in this paper.

The smallest deviations were achieved by k- $\varepsilon$ Standard model with $5.5 \%$ for the cold fraction and $6.2 \%$ for the hot fraction in a the comparison with experimental values.

This research was financially supported by the Brno University of Technology, Faculty of Mechanical Engineering in frame of the Specific Research Fund, grant no.: FSI-S-17-4531

\section{References}

1. W. Fröhlingsdorf, H. Unger, Int J Heat Mass Transf, 42, (1999)

2. N. Aljuwayhel, G. Nellis, S. Klein, Int J Refrig, 28, (2005)

3. S. Eiamsa-ard, P. Promvonge, Int J Heat Mass Transf, 50, (2007)

4. H. Skye, G. Nellis, S. Klein, Int J Refrig, 29, (2006)

5. U. Behera, P. Paul, K. Dinesh, S. Jacob, Int J Heat Mass Transf, 51, (2008)

6. T. Farouk, B. Farouk, A. Gutsol, Int J Heat Mass Transf, 52, (2009)

7. R. Shamsoddini, A. Nezhad, Int J Refrig, 33, (2010)

8. A. Ouadha, M. Baghdad, Y. Addad, Int J Refrig, 36, (2013)

9. M. Bovand, M. Valipour, S. Eiamsa-ard, A. Tamayol, Int Commun Heat Mass Transf, 50, (2014)

10. V. Alekhin, V. Bianco, A. Khait, A. Noskov, Int J Therm Sci, 89, (2015)

11. X. Liu, Z. Liu, Appl Therm Eng, 67, (2014)

12. V. Bianco, A. Khait, A. Noskov, V. Alekhin, Appl Therm Eng, 106, (2016)

13. H. Thakare, A. Parekh, Energy, 133, (2017)

14. H. Thakare, A. Parekh, Int J Heat Mass Transf, 78, (2014)

15. S. Eiamsa-ard, P. Promvonge, Int Commun Heat Mass Transf, 35, (2008) 
16. M. Baghdad, A. Ouadha, O. Imine, Y. Addad, Int J Therm Sci, 50, (2011)

17. T. Dutta, K. Sinhamahapatra, S. Bandyopdhyay, Int J Refrig, 33, (2010)

18. T. Farouk, B. Farouk, Int J Heat Mass Transf, 50, (2007)

19. A. Secchiaroli, R. Ricci, S. Montelpare, V. D 'alessandro, Int J Heat Mass Transf, 52, (2009) 